\title{
Photocurrent generation in single electron tunneling transistors
}

\author{
Ola Tageman \\ Department of Applied Physics, Chalmers University of Technology and Göteborg University \\ S-41296 Göteborg, Sweden \\ E-mail: tageman@fy.chalmers.se \\ Received October 14, 1998
}

\begin{abstract}
A single-electron tunneling transistor (SET) with a non-equilibrium mode population in one of the leads is analyzed theoretically. We model transport through a dot coupled to a channel, both formed by gates from the two-dimensional electron gas of a GaAs/AlGaAs heterostructure. The non-equilibrium mode population, which is induced by coherent THz-pumping in the channel, produces empty states below the Fermi level for electrons to tunnel into. A photocurrent arises, which is periodically saw-tooth peaked with respect to the voltage on a central gate. For intense THz-fields the peaks display plateaus that reflect the energy dependence of the mode population. We also predict a high-gain $V_{\mathrm{in}} / V_{\text {out }}$ transfer-characteristic, similar to that of a current biased SET.
\end{abstract}

PACS: $73.23 . \mathrm{Hk}$

\section{Introduction}

Charging effects are becoming increasingly important in transport experiments, as fabrication technology for nano-scale systems develops. These effects appear in different kinds of circuits that have one thing in common - they all incor porate a small semi-isolated island in which the number of electrons is strongly quantized [1-3]. At low driving voltage, fluctuations of the charge on this island are suppressed due to Coulomb interaction, except if the electrostatic potential of the island is carefully tuned [4]. This Coulomb blockade mechanism is utilized in the Single Electron Transistor (SET), to modulate the conductance through the island by varying the potential on a nearby gate electrode [5].

When alternating fields are introduced in SETs and related systems, interesting effects arise, and large amount of physical information can be gained. By applying $\mathrm{MHz}$-signals to one or several gates, one can generate precise currents in the $\mathrm{pA}$-range [6-8], and by monitoring the reflection of a microwave signal from the SET, one can follow charge fluctuations with high accuracy [9]. In these examples the alternating field acts in a quasi-static way, but if the frequency is raised further, then high-frequency effects appear. For example, if a microwave signal in the range $10-75 \mathrm{GHz}$ is fed to the gate of a planar semiconductor SET, both photon assisted tunneling across the barriers and photo-excitations inside the island, can be observed [10].

So far, no special attention has been paid to utilizing ac-fields for inducing a non-equilibrium electron distribution inside the leads of a SET. Such a distribution can clearly open new ways of tunneling into and out of the island. In a metallic lead of typical dimensions this will have a negligible effect because of strong screening and poor size-quantization. However, in a quasi one-dimensional conductor, such as a split-gate channel in the two-dimensional electron gas (2DEG) of a GaAs/AlGaAs heterostructure, the situation is different. Recent transport experiments employing $\mathrm{THz}$-fields touch upon this issue $[11,12]$.

In this work we consider, theoretically, how the performance of a SET will be influenced by the application of an electric THz-field in one of the leads. The important new ingredient is a non-equilibrium mode-population, which results in a photocurrent generation in the SET. For intense THzfields we find a short-circuit current that reveals information about the non-equilibrium distribution function in the lead. Considering instead weak $\mathrm{THz}$-fields in an open-circuit configuration, we find 
a $V_{\text {in }} / V_{\text {out }}$ transfer characteristic similar to that of the current biased SET.

\section{Theory}

The system under consideration is described in Fig. 1. We choose to have a gated-2DEG realization in mind when modeling the system. Such a realization allows both Coulomb-blockade effects and ballistic motion over many electron wavelengths $[13,14]$. When applying a negative voltage to the gates, the electrons are confined to a dot and a channel, plus to reservoirs on the two sides. We shall refer to the channel-side reservoir as the collector and to the reservoir on the other side as emitter. The emitter is grounded to a back-gate and the collector is also grounded but via a load resistor $R_{L}$. Our aim is to calculate the current $I$ through, or the voltage $V$ across this load resistor, as a function of the electrostatic potential on the dot, which can be tuned via the voltage $V_{g}$. We use the standard approach, ignore co-tunneling and calculate the current from a master equation $[15,16]$. The influence of discrete states in the dot will be ignored in order to highlight the influence of $\mathrm{THz}-$ pumping [17]. We take the band-bottom in the emitter as our reference of energy.

As long as the width of the channel varies slowly on the scale of the electron wavelength, elastic scattering between transverse modes may be ignored [18]. The resulting mode-potentials are sketched in the lower part of Fig. 1. We choose the width of the channel such that only the lowest mode enters the channel. However, by the application of a coherent electric THz-field of strength $E$, across the channel, we excite higher modes inside the channel, provided that the angular frequency $\omega$

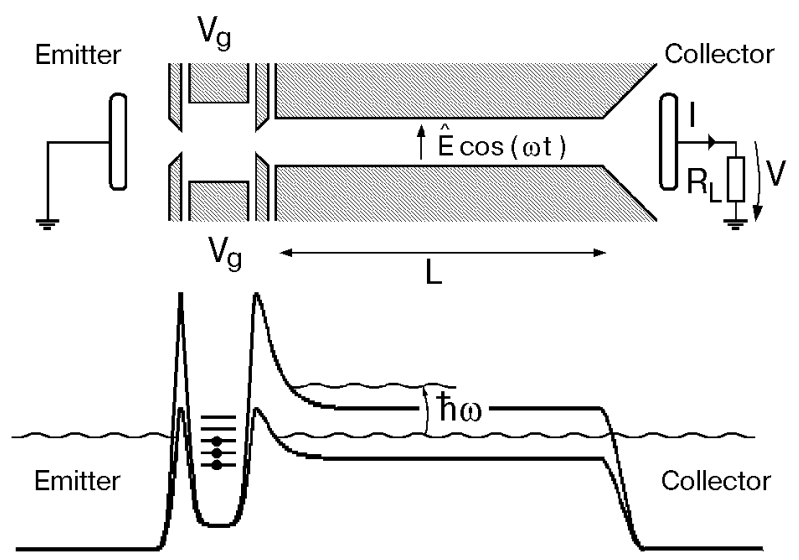

Fig. 1. Sketch of the model system and the corresponding energy diagram. A coherent electric THz-field, polarized across the collector-channel induces a non-equilibrium mode population. is chosen to match the mode-potential separation there fairly well.

We consider a situation in which, even if higher modes are brought to life by absorption of a number of energy-quanta $\hbar \omega$ from the THz-field, only the lowest mode is involved in the tunneling process. This is realistic for a split-gate induced tunneling barrier. If $\hbar \omega$ matches the mode potential separation inside the channel, the kinetic energy in an excited mode will be the same as in the lowest mode. However, at the end of the channel, where the transverse confinement is made stronger by separate split-gates, the kinetic energies will differ. The stronger confinement leads to a larger mode potential separation, as indicated in Fig. 1. Consequently, the kinetic energies decrease more in excited modes. For this reason it is realistic to assume a suppressed tunneling for excited modes.

\section{Population of collector modes}

We assume a parabolic confining potential in the channel [19]. Introducing the parameter $U_{0}$, which describes a lifting of the bottom of the well, and $\Omega$, which describes how narrow the channel is, we write

$$
U(y)=U_{0}+\frac{1}{2} m^{*} \Omega^{2} y^{2},
$$

where $m^{*}$ is the effective mass and $y$ is the transverse coordinate. The problem of finding the population of different modes in a pumped straight parabolic channel has been addressed previously [20]. It was shown that if the deviation from perfect parabolicity is sufficiently large we can forget about coupling to higher modes. We assume that this is the case and confine our interest to the lowest two modes. It is then straightforward to find an analytical solution.

Starting from the following expression (in which $e>0$ is the elementary charge) for the kinetic energy $K_{n}(E)$ in mode $n$ :

$$
K_{n}(E)=E+e V-U_{0}-\left(n-\frac{1}{2}\right) \hbar \Omega+(n-1) \hbar \omega,
$$

using the known expression [20] for the coupling energy $V_{\omega}$ :

$$
V_{\omega}=\frac{e \hat{E}}{2 \omega}\left(\frac{\hbar \Omega}{2 m^{*}}\right)^{1 / 2},
$$

and assuming that $|\hbar(\omega-\Omega)|<<K_{1}(E), K_{2}(E)$ and that $V_{\omega}<<\hbar \omega$ we get for the population $\xi(E)$ of the lowest mode at the end of the channel 


$$
\xi(E)=1-\gamma \sin ^{2}[q(E) L]
$$

Here

$$
\begin{gathered}
\gamma=\left[1+\left(\frac{\hbar(\omega-\Omega)}{2 V_{\omega}}\right)^{2}\right]^{-1}, \\
q(E)=\bar{q}(E)\left[\left(\frac{\hbar(\omega-\Omega)}{4 \bar{K}(E)}\right)^{2}+\left(\frac{V_{\omega}}{2 \bar{K}(E)}\right)^{2}\right]^{1 / 2}, \\
\bar{q}(E)=\left[\frac{2 m^{*} \bar{K}(E)}{\hbar^{2}}\right]^{1 / 2}, \\
\bar{K}(E)=\frac{K_{1}(E)+K_{2}(E)}{2},
\end{gathered}
$$

There is a strong similarity between Eq. (4) and Rabi's formula for the time developments of a two-level system. Rabi's formula describes population oscillations in time in a two-level system, while our Eq.(4) describes population oscillations between transverse modes, as a function of the spatial coordinate along the channel. The wave-vector of oscillation along the channel is given by $q(E)$ and the resonance strength by $\gamma$.

\section{Master equation approach}

By $E_{d}(E, N)$ we denote the energy, relative to the bottom of the well in the dot, of an electron that has tunneled into the dot starting from an energy $E$, when the number of electrons on the dot increases from $N-1$ to $N$. If an electron is to tunnel out of the dot into a lead at energy $E$, thereby decreasing the number of electrons from $N$ to $N-1$, it must start at this very same energy $E_{d}(E, N)$. We use the following capacitance model for relating $E_{d}(E, N)$ to $E$ :

$E_{d}(E, N)=E-N \frac{e^{2}}{C_{\Sigma}}+e V_{g} \frac{C_{g}}{C_{\Sigma}}+e V \frac{C_{c}}{C_{\Sigma}}+$ const .

Here $C_{\Sigma}$ is the total capacitance of the $\operatorname{dot}$; $C_{g}$ is the capacitance between the gate electrodes and the $\operatorname{dot}$; $C_{c}$ is the capacitance between the collector and the dot. The electrostatic potential of the dot can be continuously tuned via the gate potential $V_{g}$ and it is dependent also on the electrostatic potential $V$ in the collector. In addition there are contributions to the potential energy on the dot from the point contact gates and from the rest of the surroundings, in particular from random offset charges. We treat this as an unknown constant in Eq. (6). It corre- sponds to a shift in $V_{g}$, which we shall not try to determine.

We assume that the electrons inside the dot, after each tunneling event, quickly thermalize to a Fermi-Dirac distribution with a temperature $T$ given by the surrounding and with a chemical potential $\mu(N)$ given by the number of electrons inside the dot. It then makes sense to introduce $P(N)$, the probability for having $N$ electrons on the dot. Furthermore, we can use $f\left[E_{d}-\mu(N)\right]$ for the probability of finding an electron in a single-electron energy-level $E_{d}$, given that there are $N$ electrons on the dot, where

$$
f(x)=\left[\mathrm{e}^{x / k T}+1\right]^{-1} .
$$

By assuming that $k T>\Delta E$ we are allowed to use a continuum description in the dot, and we take the density of states there, $\rho_{d}$, to be constant. Moreover, we assume that $\Delta E<<e^{2} / C_{\Sigma}$ and therefore ignore the variation of $\mu(N)$ with $N$. For simplicity we assume that $\mu$ is so large $\left(>E_{F}-U_{0}\right)$ that its actual value has no influence other than adding to the constant in Eq. (6).

The probability distribution $P(N)$ is found from the following balance equation:

$$
\begin{array}{r}
\quad P(N)\left[W_{e \rightarrow d}(N+1)+W_{c \rightarrow d}(N+1)\right]= \\
=P(N+1)\left[W_{d \rightarrow e}(N+1)+W_{d \rightarrow c}(N+1)\right]
\end{array}
$$

plus the normalization condition

$$
\sum_{N} P(N)=1 .
$$

In Eq. (9) we have used the following definitions of the total tunneling rates: by $W_{e \rightarrow d}(N)$ we denote the rate at which electrons tunnel from the emitter into the dot when there are $N-1$ electrons on the dot to start with and thus $N$ electrons on the dot in the final state; by $W_{d \rightarrow e}(N)$ we denote the rate for the reversed process, in which electrons leave the dot when there are $N$ electrons on it to start with; we denote the corresponding collector rates by $W_{c \rightarrow d}(N)$ and $W_{d \rightarrow c}(N)$.

The current $I$, as defined in Fig. 1, can be found by summing the rates for tunneling out of and into the emitter:

$$
I=-e \sum_{N} P(N)\left[W_{e \rightarrow d}(N+1)-W_{d \rightarrow e}(N)\right] .
$$

\section{Tunneling rates}

The barriers are formed by split gates and the voltage on these gates is held constant. We crudely 
choose to ignore the dependence of the barriers on both the photovoltage $V$ and the number of electrons on the $\operatorname{dot} N$. We start from golden-rule expressions for the tunneling rates. By $\Gamma_{e}(E)$ we denote the rate at which electrons leave the emitter at an absolute energy $E$ and tunnel into the dot. The same rate is used for the reverse process. By $\Gamma_{c}(E)$ we denote the corresponding tunneling rates from and into the collector,

$$
\Gamma_{e / c}(E)=\frac{2 \pi}{\hbar}\left|M_{e / c}(E)\right|^{2} \rho_{d} .
$$

Here $M_{e}(E)$ and $M_{c}(E)$ are tunneling matrix elements which are given by some overlap integral between initial and final states. Since we are not interested in the details in these matrix elements we choose to describe tunneling by a resistance $R_{e}$ for the emitter barrier and a resistance $R_{c}$ for the collector barrier. Both resistances are defined at the Fermi-level.

However, the tunneling resistances $R_{e}$ and $R_{c}$ are energy dependent. In our case this dependence must be modeled because the pumping mechanism will depopulate the collector and allow for tunneling far below $E_{F}$. We assume an exponential decay in the tunneling rates when the energy is lowered and we introduce a tunneling-decay scale $E_{T}$ for this purpose. The following form is used for the matrix elements:

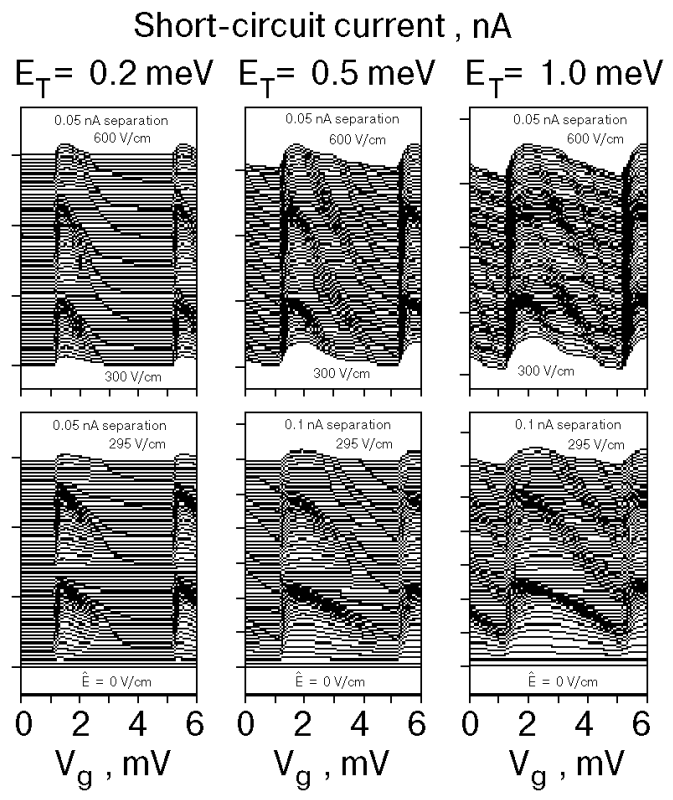

Fig. 2. Short-circuit current $-I\left(V_{g}\right)$ for three different values of the tunneling-decay scale $E_{T}$. Each curve corresponds to a particular value of the field strength $\hat{E}$. For clarity the curves are separated by a constant offset.

$$
\left|M_{e / c}(E)\right|^{2}=\frac{\hbar \mathrm{e}^{\left(E-E_{F}\right) / E_{T}}}{2 \pi e^{2} \rho_{d} \rho_{e / c} R_{e / c}},
$$

where $\rho_{e / c}$ is the density of states in the emitter/collector, which we take to be constant. With this choice all densities of states cancel and we instead introduce the tunneling resistances, which can be measured in an experiment by opening one point contact at a time.

The total rates are given by sums of partial rates associated with the different energies. Turning to a continuum description we get

$$
\begin{gathered}
W_{e \rightarrow d}(N)=\int_{0}^{\infty} \rho_{e} f\left(E-E_{F}\right) \times \\
\times \Gamma_{e}(E)\left\{1-f\left[E_{d}(E, N)-\mu\right]\right\} d E, \\
W_{d \rightarrow e}(N)=\int_{0}^{\infty} \rho_{e} f\left(E_{d}(E, N)-\mu\right) \times \\
\times \Gamma_{e}(E)\left[1-f\left(E-E_{F}\right)\right] d E, \\
W_{c \rightarrow d}(N)=\int_{c}^{\infty} \rho_{c} f\left(E+e V-E_{F}\right) \xi(E) \times \\
U_{0}+\hbar \Omega / 2-e V \\
\times \Gamma_{c}(E)\left\{1-f\left[E_{d}(E, N)-\mu\right]\right\} d E, \\
W_{d \rightarrow c}(N)=\int_{U_{0}+\hbar \Omega / 2}^{\infty} \rho_{c} f\left[E_{d}(E, N)-\mu\right] \times \\
\times \Gamma_{c}(E)\left[1-f\left(E+e V-E_{F}\right) \xi(E)\right] d E .
\end{gathered}
$$

\section{Short-circuit current}

When $R_{L}=0$ we know that $V=0$ and we can find the current from Eq. (10). In our numerical calculations we vary three important experimental variables, $V_{g}, \hat{E}$ and $E_{T}$. For the rest of the parameters we use the following realistic values: $R_{e}=R_{c}=200 \mathrm{k} \Omega, C_{\Sigma}=100 \mathrm{aF}, C_{g}=0.4 C_{\Sigma}, T=$ $=0.1 \mathrm{~K}, E_{f}=14 \mathrm{meV}, U_{0}=10 \mathrm{meV}, \hbar \Omega=4 \mathrm{meV}$ and $L=2.5 \mu \mathrm{m}$. We assume that the frequency, or equivalently the voltage on the gates that form the channel, is tuned to resonance, i.e., $|\hbar(\omega-\Omega)|<<$ $<<2 V_{\omega}$ [see $\gamma$ in Eq. (5)]. 
With our definition in Fig. 1 the current $I$ is negative. In order to avoid confusion in our explanations we shall always plot $-I$. In Fig. 2 we plot $-I$, as a function of $V_{g}$, for a set of field strengths $\hat{E}$ ranging from 0 to $500 \mathrm{~V} / \mathrm{cm}$ for three different values of the tunneling-decay scale $E_{T}: 0.2,0.5$, and $1.0 \mathrm{meV}$. Upon increasing $V_{g}$, at some point $-I$ increases abruptly and then decays more slowly, until the next point of increase. For large $E_{T}$ plateaus are seen on the decaying side, especially when $E$ is large.

We explain this behavior in Fig. 3. An enlargement of one of the curves is shown to the left, the one with $E_{T}=0.5 \mathrm{meV}$ and $\hat{E}=600 \mathrm{~V} / \mathrm{cm}$. In the right part we illustrate the population of both charge-states and lead-states, corresponding to the cross-hair point on the current curve. Because of the pumping, depopulation appears in the collector which means that not all levels below the Fermilevel are occupied. Such depopulation enables tunneling out of the dot.

Increasing $V_{q}$ corresponds to lowering all charge-states in the dot. The dramatic increase in $-I$ occurs when a charge state falls below $E_{F}$ in the emitter so that there is a way to fill the dot. To start with the inflow from the emitter limits $-I$, and therefore $-I$ rises in proportion to the lowering of this charge state.

After the top, the outflow will limit $-I$. The slow decay of the current is understandable since the outflow takes place at many different energies and we must thus bring the charge-state far down in order to shut $-I$ off completely. The tunnelingdecay scale $E_{T}$ determines this decay rate.

Also, the plateaus in the decay can be understood from the population diagram. The first plateau appears when the charge state passes the first population maxima as shown to the right in Fig. 3. Since the charge-state is occupied most of the time, the outflow rate from this charge-state limits the $-I$. But at the population maxima there are no empty states to tunnel into, which means that the outflow is insensitive to a change in $V_{g}$ there. The reason why the plateau is not perfectly flat, is that as we pass a population peak there is an increasing backflow from the collector, which tends to reduce the net flow.

As we continue increase $V_{g}$ and thus lower the charge-states, $-I$ drops when we pass population minima and is steady when we pass a population maximum in a repetitive manner. The steps get smaller and smaller as the energy-period of population oscillations decreases.

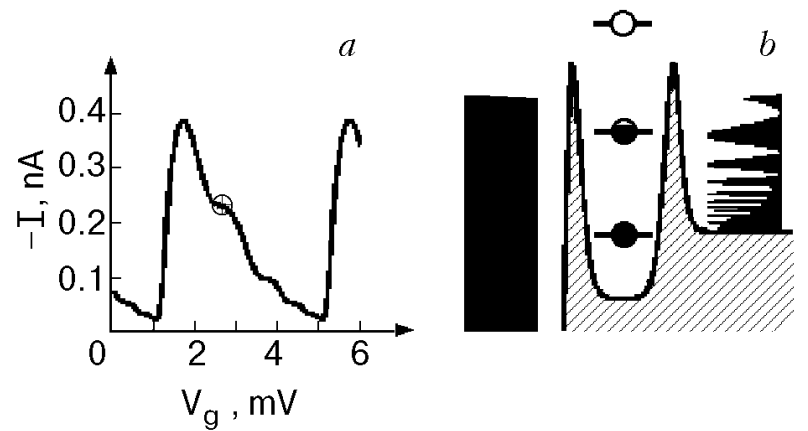

Fig. 3. Short-circuit current $-I\left(V_{g}\right)$ for the $E_{T}=0.5 \mathrm{meV}$ and $\hat{E}=600 \mathrm{~V} / \mathrm{cm}($ a $)$ and population diagram corresponding to the cross-hair point $(b)$. The energy dependence in the population of collector levels gives rise to plateaus in $-I\left(V_{g}\right)$. Note that a population peak is aligned with a charge state for this particular choice of $V_{g}$

\section{Voltage gain}

In this section we deal with photovoltaic effects. We now assume the collector load $R_{L}$ to be in the $\mathrm{M} \Omega$-range, allowing a significant negative collector-potential $V$ to build up relative to the grounded emitter. The load $R_{L}$, which simulates either a voltmeter or the input of another transistor, is varied in order to demonstrate the driving capability of the device. We must now solve for the particular value of $V$ that gives rise to a current $I$ given by Eq. (10) that fulfills $V / I=R_{L}$

We make two assumptions about the system that must be fulfilled in an experiment, if the voltage swing is not to be limited. First, we assume that the mode spectrum in the channel is robust to changes in the channel-gate potential. If it is not, the system is brought out of resonance as $V$ changes, which makes the pumping ineffective. In the Appendix we elaborate a bit more on this point. Second, we assume that the tunneling barriers are relatively high and thin and allow for tunneling far below the top. To this end we set $E_{T}=0.5 \mathrm{meV}$ and $R_{e}=R_{c}=1 \mathrm{M} \Omega$. The high values of the tunneling resistances assure that the charge-states are well quantized even when $V$ rises. A promising technique for achieving high barriers is Inplane gating [21].

Figure 4 shows the transfer characteristic $-V\left(V_{g}\right)$, for $\hat{E}=40 \mathrm{~V} / \mathrm{cm}$, when $C_{g}=0.4 C_{\Sigma}$ and $C_{c}=0.1 C_{\Sigma}$. Note that we plot $-V$, which is positive, in order to avoid confusion in the explanations. The result is not very sensitive to a variation of $\hat{E}$. Each curve corresponds to a particular value of $R_{L}$. The voltage gain, which can be read off as the steepest slope of a curve, is ideally $C_{g} / C_{c}$. This can be understood from Fig. 5, $a$, where we analyze the rising edge for the case $R_{L}=1000 \mathrm{M} \Omega$. 


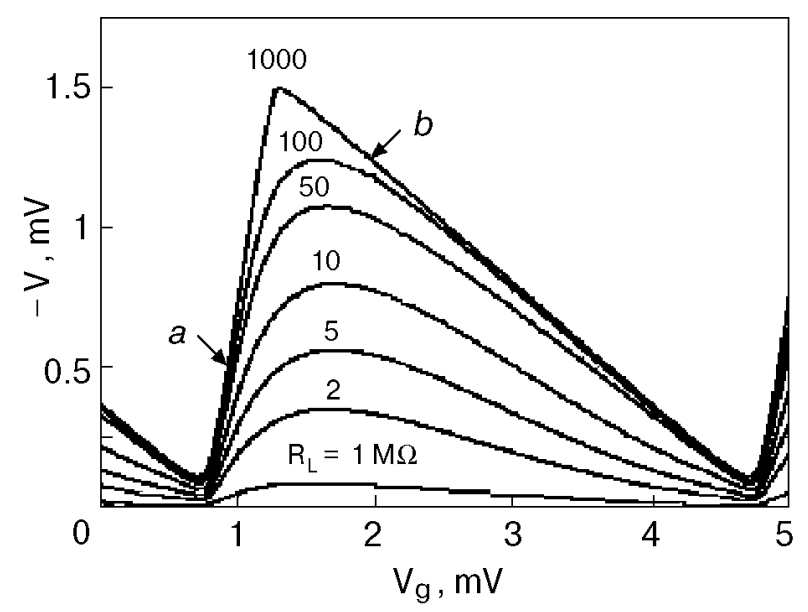

Fif. 4. Transfer-characteristic, when $\hat{E}=40 \mathrm{~V} / \mathrm{cm}$, for different values of the load resistance. For $R_{L}=1000 \mathrm{M} \Omega$ the voltage gain exceeds 3 , but it smaller for smaller $R_{L}$.

Let us for the moment put $T=0, R_{L}=\infty$ and ignore all but one charge-state. When this charge state is exactly leveled with $E_{F}$, in the emitter the current is blocked since there can be no flow between the emitter and the charge-state. However, when $V_{g}$ is slightly raised the charge-state is lowered via capacitive coupling. Now electrons start to flow from the emitter into the charge-state. The flow from the charge-state into the collector is already made possible due to the depopulation in the collector. Since the collector is an isolated region, such a flow will accumulate electrons on the collector, and accordingly its electrostatic potential $V$ will become more negative. Because the dot is also coupled capacitively to the collector, the decrease in $V$ tends to lift the charge-state back again. This accumulation stops when the charge-state again is leveled with $E_{F}$. This way the electrostatic potential of the dot is regulated to be constant, and from Eq. (6) it is then clear that $\partial V / \partial V_{g}=$ $=-C_{g} / C_{c}$. In reality there will be corrections to this simple description that reduces the voltage gain. An estimation of the maximum slope of the $R_{L}=1000 \mathrm{M} \Omega$ curve in Fig. 4 indicates that the gain is about 3.3 and not exactly 4 .

The falling edge in $-V\left(V_{g}\right)$ is governed by a different mechanism. As illustrated in Fig. 5,b, a strong back-flow sets in when $E_{F}$ in the collector tries to rise above the next higher charge-state. This back-flow drains the collector of electrons, and $E_{F}$ in the collector is thus bound to follow the higher charge-state on its way down. By a consideration similar to that for the rising edge we find a slope: $\partial V / \partial V_{g}=-C_{g} /\left(C_{\Sigma}-C_{c}\right)$.
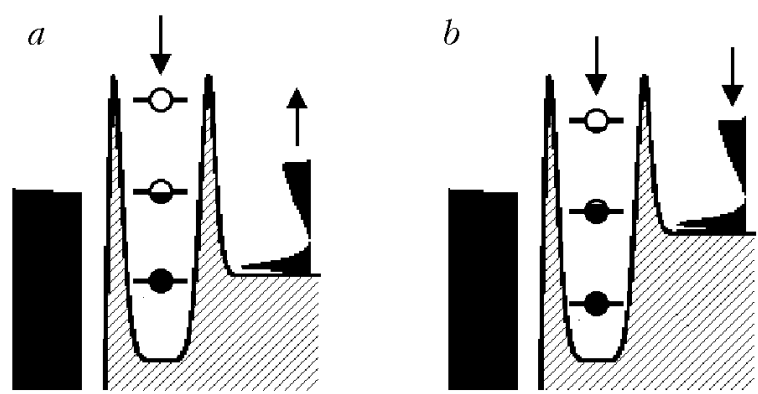

Fig. 5. Level diagrams for two different points on the transfercharacteristic for $R_{L}=1000 \mathrm{M} \Omega$. In a) the mechanism behind the rising edge of $-V$ is illustrated. A charge state is aligned with $E_{F}$ in the emitter. When $V_{g}$ is raised slightly the charge state is lowered via $C_{g}$ and $V$ starts to decrease. This decrease in $V$ tends to lift the charge state back again via $C_{c}$. Ideally the gain is $C_{g} / C_{c}$. In b) the mechanism behind the falling edge of $-V$ is illustrated. Here $E_{F}$ in the collector has reached a higher charge state. Now a lowering of the charge states is allowed.

\section{Discussion}

An interesting aspect of our findings is that not only Fermi-level properties are probed. The plateaus in the short-circuit current, reflect the energy dependence of the mode-population. If a plateau can be observed for some energy below the Fermilevel, then we know that a single particle description without phase-breaking works well at that energy. The influence of phase breaking is most pronounced for slow electrons since they spend more time inside the channel. Therefore, we expect the smallest plateaus in the short-circuit current to disappear first. There is room for future work on how to account for phase-breaking.

The photovoltage result of section 4 seems less sensitive to the details in the depopulation and it requires a comparatively small field-strength of the THz-field [11]. Photovoltage generation relies in principle only on there being depopulated states in the collector for electrons to tunnel out to. Even though the precise energy-dependence of the depopulation determines the current-driving capability, it does not have much influence on the open-circuit voltage.

The transfer characteristic for large $R_{L}$ in Fig. 4 is very similar to that of the current biased SET with a large load resistance [22]. In particular it has the same voltage gain, and the underlying mechanisms are similar. This kind of transfer characteristic seems attractive from the application perspective since it allows for poor precision in fabrication of cascaded devices for logic applications. It has good gain, large voltage-swing and shows a saturation-like behavior. Such properties in combi- 
nation make the precise value of the $V_{g}$-threshold, within limits, irrelevant.

A pumped SET has the potential of giving a very simple circuit architecture. Each device works as a tiny voltage-controlled battery, which takes its energy from the THz-field. There is thus no need for power-lines on a chip. Nor do we need clock-signal lines since in principle we can synchronize the devices by modulating the frequency of the THzfield. A similar vision has been presented for devices consisting of chains of islands [23].

Future research on material science and fabrication technology may bring other ways of realizing a pumped SET than the one we have in mind. The key ingredient is a channel, adiabatically connected to reservoirs, in which the electrons preserve their coherence for 50 wavelengths or so. It would be advantageous to define the boundaries of the device without metallic gate electrodes, using for example etching and regrowth techniques, focused ion-beam implantation or perhaps - many years from now by tailoring a carbon nanotube [3,24,25].

\section{Conclusions}

We have analyzed the influence of THz-pumping in one of the leads of a SET. THz-induced depopulation opens the possibility for electrons to leave the dot below the Fermi-level allowing a photocurrent to flow. As the gate voltage is changed, the charge state in the dot acts as a probe of the energy dependence of this depopulation. From the application perspective we present a new way of obtaining voltage-gain from a SET.

\section{Appendix}

If the walls of the channel move as the electrostatic potential in the collector changes relative to that in the channel-gates, it may result in a limited output voltage swing. From Eq. (7) we find that the system is brought out of resonance if the modespacing $\hbar \Omega$ changes by more than $2 V_{\omega}$. In a typical experiment [26] it takes a change in the split-gate voltage of about $100 \mathrm{mV}$ to go from the threshold of mode 1 to the threshold of mode 2. Assuming a parabolic potential and considering the worst case, in which $U_{0}$ does not change at all, we find that this corresponds to a $40 \%$ change in the mode-spacing $\hbar \Omega$. From this we conclude that if the modespacing is allowed to change only by an amount $2 V_{\omega}$ then the voltage swing must not exceed $-V_{\max }^{\omega}=(100 \mathrm{mV}) 2 V_{\omega} / 0.40 \hbar \Omega$. With our choice of parameters in section 4 , we get: $-V_{\max }==3 \mathrm{mV}$. Thus, we can not rule out such an influence in a
gated-2DEG realization of the system. To get good performance it may be necessary to look for a different fabrication technique, for example an etching and regrowth technique [24].

\section{Acknowledgments}

We acknowledge financial support from the EU (MEL ARI Research project 22953 - CHARGE), from the Swedish Research Council for Engineering Sciences (TFR) and from Ericsson Microwave Systems AB. We are greatful to L. Y. Gorelik who proposed the problem and who has contributed in discussion. Also Peter Wahlgren and Mats Jonson are acknowledged for helpful discussions.

1. T. A. Fulton and G. J. Dolan, Phys. Rev. Lett. 59, 109 (1987).

2. J. H. F. Scott-Thomas, S. B. Field, M. A. Kastner, H. I. Smith, and D. A. Antoniadis, Phys. Rev. B62, 583 (1989).

3. S. J. Tans, M. H. Devoret, H. Dai, A. Therss, R. E. Smalley, L. J. Geerligs, and C. Dekker, Nature 386, 474 (1997).

4. I. O. Kulik and R. I. Shekhter, Zh. Eksp. Teor. Fiz. 68, 623 (1975), [Sov. Phys. -JETP 41, 308 (1975)].

5. K. K. Likharev, IEEE Trans. Magn. 23, 1142 (1987).

6. L. J. Geerligs, V. F Anderegg, P. A. M. Holweg, and J. E. Mooij, Phys. Rev. Lett. 64, 2691 (1990).

7. H. Pothier, P. Lafarge, C. Urbina, D. Esteve, and M. H. Devoret, Europhys. Lett. 17, 249 (1992).

8. J. M. Martinis, M. Nahum, and J. D. Jensen, Phys. Rev. Lett. 72, 904 (1994).

9. R. J. Schoelkopf, P. Wahlgren, A. A. Kozhevnikov, P. Delsing, and D. E. Prober, Science 280, 1238 (1998).

10. T. H. Oosterkamp, L.P. Kouwenhoven, A. E. A. Koolen, N. C. van der Vaart, and C. J. P. M. Harmans, Phys. Rev. Lett. 78, 1536 (1997).

11. D. D. Arnone, J. E. F. Frost, C. G. Smith, D. A. Ritchie, G. A. C. Jones, and R. J. Butcher Appl. Phys. Lett. 66, 3149 (1995).

12. Q. Hu. S. Verghese, R. A. Wyss, Th Schäpers, J. del Alamo, S. Feng, K. Yakubo, M. J. Rooks, M. R. Melloch, and A. Förster, Semicond. Sci. Technol. 11, 1888 (1996).

13. L. P. Kouwenhoven, N. C. van der Vaart, A. T. Johnson, W. Kool, C. J. P. M. Harmans, J. G. Williamson, A. A. M. Staring, and C. T. Foxon, Z. Phys. B - Cond. Matter. 85, 367 (1991).

14. T. Honda, S. Tarucha, T. Saku, and Y. Tokura Jpn. J. Appl. Phys. 34, L72 (1995).

15. C. W. J. Beenakker, Phys. Rev. B44, 1646 (1991).

16. D. V. Averin, A. N. Korotkov, and K. K. Likharev, Phys. Rev. B44, 6199 (1991).

17. A. T. Johnson, L. P. Kouwenhoven, W. de Jong, N. C. van der Vaart, and C. J. P. M. Harmans, Phys. Rev. Lett. 69, 1592 (1992).

18. L. I. Glazman, G. B. Lesovik, D. E. Khmel'nitskii, and R.I. Shekhter, Pisma Zh. Eksp. Teor. Fiz. 48, 258 (1988) [JETP Lett. 48, 238 (1988)].

19. S. E. Laux, D.J. Frank, and Frank Stern, Surf. Sci. 196, 101 (1988).

20. Ola Tageman and L.Y. Gorelik, J. Appl. Phys. 83, 1513 (1998). 
21. H. Tomozawa, K. Jinushi, H. Okada, T. Hashizume, and H. Hasegawa, Physica B227, 112 (1996).

22. M. I. Lutwyche and Y. Wada, J. Appl. Phys. 75, 3654 (1994).

23. A. N. Korotkov, Appl. Phys. Lett. 67, 2412 (1995).

24. P. Ramvall, N. Carlsson, I. Maximov, P. Omling, L. Samuelsson, W. Seifert, Q. Wang, and S. Lourdudoss, Appl. Phys. Lett. 71, 918 (1997).
25. T. Fujisawa, Y. Hirayama, and S. Tarucha, Appl. Phys. Lett. 64, 2250 (1994).

26. B. J. van Wees, H. van Houten, C. W. J. Beenakker, J. G. Williamson, L. P. Kouwenhoven, D. van der Marel, and C. T. Foxon, Phys. Rev. Lett. 60, 848 (1988). 